\title{
Voir ce que je vois, voir ce que je m'attends à voir : degré zéro de l'écriture du voyage et écriture littéraire du voyage. En citant Henri Michaux et Michel Butor
}

\author{
JEAN BESSIÈRE
}

\begin{abstract}
To see what I expect to see: travel writing's degree zero and literary travel narratives. With references to Henri Michaux and Michel Butor. This essay examines what arguments can be put forward to explain why readers and critics view travel writing as literary. It offers an answer that does not imply any coded definition of literature and literary works: literary travel writing is the mimesis of the questioning which characterises any literary work. This questioning rests on:
\end{abstract}

1. The duality of travellers' perceptions of the foreign lands they discover. They see what they see and what they expect to see; their perceptions are mediated and unmediated, and consequently reflexive and congruent with the cognitive undecipherability of the foreign lands.

2. The paradox of the situation of the traveller/writer. Abroad, the traveller is not viewed as a foreigner; the least difference he/she embodies highlights a paradoxical cognitive undecipherability. The effect of the auctorial enunciation is limited by this paradox.

3. The reflexive construction of the piece of travel writing. Because they bar any meta-description of the foreign land and its people, the duality of perceptions and the traveller's paradox make the evocations of places and people at once autonomous and implicitly related.

4. The behaviourist approach to the people of the foreign land(s). These restrictions to the traveller's power to interpret makes the behaviourist approach obligatory. People of foreign lands can be viewed as objective entities.

5. The implicit inferences that human objective entities motivate and suggest an overall questioning.

These critical and theoretical views utilise references to Michaux's and Butor's travels abroad and their travel writing.

Keywords: Blaise Cendrars; Michel Butor; travel; literary writing; intentionality; questioning 
BESSIÈRE

L'écriture du voyage est innombrable, ainsi que le sont ses types, comme le sont les types de voyages. Les écrivains restent des praticiens remarqués de cette écriture. L'essentiel n'est pas ici de souligner que le compte rendu de voyage est reconnu comme appartenant, de plein droit, à la littérature, mais d'analyser comment voyage et écriture du voyage, venue d'écrivains, s'éclairent mutuellement, et imposent ces questions : en quoi la pratique du voyage et son écriture permettent-elles une identification de l'écriture littéraire per se ? En quoi celleci permet-elle le mouvement inverse, l'approche du voyage per se - soit le critique suppose qu'une telle approche stricte du voyage est possible, soit il estime qu'elle aide à dessiner une heuristique du voyage. Bien évidemment, à l'inverse de ces notations, beaucoup aujourd'hui pensent que littérature et voyage sont indissociables et constituent un ensemble inaltérable, d'autant plus assuré que tout écrivain un peu établi parcourt le monde afin de promouvoir son œuvre et qu'il ne peut en conséquence dissocier voyage et littérature : le voyage serait la traduction de la vocation littéraire ${ }^{1}$.

Cette interrogation réciproque du voyage et de la littérature n'est pas une interrogation arbitraire. D'une part, elle repose sur le fait que le compte rendu de voyage, devenu écriture littéraire ${ }^{2}$, met en évidence la dualité de la perception du voyageur, se trouve soumis, par cette dualité, à un jeu réflexif, et présente un questionnement. D'autre part, cette interrogation du voyage et de la littérature repose sur les paradoxes qui définissent l'écrivain et son écriture. Paradoxe de l'écrivain: dans le voyage, ce n'est pas la terre parcourue qui est étrangère, mais l'écrivain. Paradoxe de l'écriture : celle-ci est l'évocation de l'étranger, inaltérablement lui-même, mais aussi l'évocation de l'écrivain, à la fois assuré de son identité, fût-ce seulement celle de son statut d'écrivain, et comme d'une identité équivoque - il est cet étranger, donc celui qui ne peut s'en remettre entièrement à son identité, dans une terre qui n'est d'abord qu'elle-même. Bien évidemment, le double jeu réflexif auquel est soumise, dans le compte rendu de voyage, la terre étrangère, fait inévitablement venir aux paradoxes de l'écriture, de l'écrivain.

1 Ce type d'attitude est aujourd'hui illustré en France par la manifestation annuelle des «Étonnants voyageurs » à Saint-Malo, créée par un écrivain voyageur, Michel Le Bris.

2 Écriture littéraire : écriture d'écrivains qui ne se définissent pas essentiellement comme des voyageurs et qui font du voyage un objet de leurs pratiques scripturaires. Le caractère littéraire de cette écriture sera ici précisé pour lui-même. 
I. De la dualité, de l'écriture du voyage et de leurs illustrations : Michaux et Butor

Il est un moyen de préciser ces remarques. On a distingué, dans la part de l'histoire du voyage, qui va de la fin du $\mathrm{XIX}^{\mathrm{e}}$ siècle, moment de l'industrialisation du tourisme, à l'époque contemporaine, le voyageur et le touriste. Le premier voit ce qu'il voit ; le second voit ce qu'il s'attend à voir. Cette distinction, largement reprise aujourd'hui, fait apparaître le touriste comme une figure dominante. Bien des figures sont opposables à celle du touriste - ainsi de celle de l'anthropologue et du privilège accordé à ce dernier (Augé, 2003) ${ }^{3}$. Cette distinction du voyageur et du touriste reste cependant incertaine. Bien des voyageurs voient ce qu'ils s'attendent à voir, sans être pour autant identifiables à des touristes. Les touristes, donc confondus avec les seules tentations et attentes de voir ce qu'il leur est donné à voir, voient inévitablement ce qu'ils voient, même si ce qu'ils voient ainsi correspond à ce qu'ils s'attendaient à voir. Certains préparent leurs voyages selon cette dualité et selon l'équilibre de ses deux parties (Botton, 2002) ${ }^{4}$. La distinction du voyageur et du touriste importe donc moins en elle-même que parce qu'elle renvoie à la dualité des voyages : d'une part, l'immédiateté du regard - voir ce qu'on voit - qui conduit à une observation behaviouriste ; d'autre part, la médiation du regard par ce que celui-ci suppose. Cette dualité est constitutive de l'écriture littéraire du voyage, et exposée dans les jeux de réflexivité et les paradoxes, précédemment notés.

Cette dualité, voir ce qu'on voit et voir ce qu'on s'attend à voir, exemplairement exposée par les écritures littéraires du voyage, caractérise toute approche de l'altérité, anthropologique, sociale, culturelle et même naturelle la reconnaissance de la nature est nécessairement culturelle. Cette approche est immédiate - elle repose sur la perception. Elle est indissociable de constructions, celles qui définissent celui qui voit, celles qui définissent celui/cela qui est vu. L'écriture du voyage expose cette dualité, l'implication de ces constructions. Il y a là son degré zéro, lorsque le voyage est parcours du monde, hors d'une finalité explicite qui prévaudrait - quête, exploration, connaissance -, lorsqu'il est comme l'exercice d'une passion spatio-temporelle à travers le changement de lieu, la brève alternance des séjours, et cependant hors de toute absorption dans l'espace.

3 Thématique illustrée par Marc Augé dans L'impossible voyage. Le tourisme et ses images (Augé 1997) et Le temps en ruines (Augé 2003) - dans ce dernier ouvrage, voir en particulier le chapitre « Tourisme et voyage, paysage et écriture ».

4 Voir une méditation sur le voyage et sa préparation, voir Alain de Botton, The Art of Travel (Botton 2002). La table des matières de l'ouvrage est, à elle seule, l'exacte présentation du voyage calculé selon son possible agrément. 
BESSIÈRE

Disons, pour illustrer ces remarques, Michaux et Butor, leurs voyages, et leurs écritures littéraires du voyage, celles d'écrivains tenus aujourd'hui pour canoniques. Michaux et Butor ont souvent vu ce qu'ils voulaient voir, ce qu'ils s'attendaient à voir, sans pour autant cesser de voir ce qu'ils voyaient comme en une manière de lien direct. Cela se lit sans détour dans les titres de leurs comptes rendus de voyage. Michaux : par le terme de barbare, le titre du compte rendu de son voyage en Asie, Un barbare en Asie (Michaux 1933), indique la radicale extériorité du voyageur et l'inévitable du fait que celui-ci voit ce qu'il voit, d'une part, et, d'autre part, que sa vision, par cette extériorité, est d'une caractérisation personnelle et culturelle spécifique : l'extériorité implique des attentes - en particulier, celle de l'autre ; ces attentes impliquent nécessairement leurs propres définitions. Butor : celui-ci offre deux titres ambivalents. Ainsi du compte rendu intitulé Où (avec l'accent grave du ù barré) (Butor 1971), et sous-titré Le génie du lieu 2. Ainsi de cet autre compterendu, intitulé Boomerang (Butor 1978) et sous-titré, Le génie du lieu 3. Les sous-titres font comprendre que ces comptes rendus peuvent se lire comme des reconnaissances plénières des lieux autres, comme leurs caractérisations complètes, à l'occasion du voyage. Le génie du lieu définit le lieu considéré en lui-même suivant ses caractères propres, d'abord identifiables dans l'espace (Butor 1958) ${ }^{5}$. Les titres font entendre : le lieu propre, pleinement reconnu, n'est qu'un lieu alternatif, substitut d'un autre lieu et lui-même objet d'une possible substitution $(O \dot{u})$, qui est à l'initiative du voyageur ; ce même lieu propre est indissociable d'un jeu de retour - ce lieu est ainsi inévitablement relativisé, éloigné de sa perception directe. Remarquons encore à propos du titre Boomerang : le boomerang est un objet culturel particulier, qui suppose une vision directe; il n'exclut pas cependant sa citation à valeur généralisante, celle du retour.

\section{De la phénoménologie du voyage}

Défaut d'absorption dans les espaces autres et alternances des lieux de séjour définissent une phénoménologie du voyage, congruente avec la dualité du voir ce qu'on voit, voir ce qu'on s'attend à voir. Allons de cette phénoménologie au degré zéro de l'écriture littéraire du voyage.

Cela est l'objet du livre de Michel Butor, Le génie du lieu, évocation plénière de quatre lieux, connus à l'occasion de voyages, sans que cette évocation appartienne à l'écriture du voyage - le voyageur n'est jamais cité (Butor 1958). 
Cette phénoménologie est celle du voyage comme il vient, segmenté, sans aveu de plan ; ses brèves alternances de séjour donnent un égal droit de cité aux voyageurs et aux lieux sans que ni les uns, ni les autres ne prévalent.

Par son titre « un barbare en Asie », Michaux fait entendre cette égalité, d'une part, son identité, d'autre part, celle de l'espace autre. Nous sommes hors du voyage dans l'empire ; nous sommes hors d'une sorte de poids de la défamiliarisation qui pèserait sur le voyageur : celui-ci conserve son identité et ne considère aucun exotisme en lui-même ; dans son évidence, l'autre expose ses scènes familières, ses propres événements, tout cela qu'il faut constater, une culture et sa disparité. De plus, les alternances de lieux font droit à l'espace autre et excluent que le voyageur s'y trouve absorbé et lui impose un ordre. Ainsi, Un barbare en Asie est-il l'évocation d'une série de pays, de lieux, de scènes de choses vues, sans que cette série porte en elle-même un argument continu chaque segment de la série et ses données valent pour eux-mêmes. Cette série et le défaut d'argument laissent entiers, dans la mesure où rien des lieux n'apparaît faire loi, l'identité du voyageur, le pouvoir de son regard et de son énonciation. Remarquablement, les fortes réactions négatives et parfois positives de Michaux face à l'Asie, qui lui ont été souvent reprochées et qu'il a regrettées, sont les preuves de cette égalité du lieu et du voyageur étranger ${ }^{6}$. C'est parce que le lieu est pleinement reconnu qu'il est l'objet de descriptions ambivalentes ; c'est parce qu'il apparaît indifférent, par sa réalité plénière, à la présence du voyageur étranger, que celui-ci peut à la fois le dire personnellement, et en reconnaître les évidences.

Les titres de Michel Butor notent l'alternance des lieux et indiquent que les séries et de leurs évocations segmentées sont librement recomposables. Dans $O \dot{u}$, chacun des chapitres correspond à un lieu, se lit de manière indépendante, et porte ses propres variations. Seul le premier chapitre, «J'ai fui Paris » a une fonction au regard de la série : il introduit celle-ci. Une constante est manifeste : les références aux moments de la journée, aux faits climatiques sont des indices du quotidien, mais aussi de ce que fait le lieu autre : avoir son évidence, sa spécificité et offrir comme une médiation au voyageur, précisément ces moments, ces faits qui sont de tout lieu. Il y a là la certitude que puisse s'exercer le regard de l'étranger : celui-ci est inséré (et non capté) dans un lieu qui a des traits génériques familiers et des traits propres. Boomerang amplifie ce jeu de la série. Des titres qui apparaissent seulement dans la table des matières et qu'on ne peut jamais lire dans corps de l'œuvre - « Jungle », « La fête en

6 Simon Leys a noté et regretté les remords de Michaux et les modifications que celui-ci a apportées aux dernières éditions d'Unbarbare en Asie. L'accusation d'orientalisme (au sens d'Edward Said) est fréquemment portée contre l'écrivain (Leys 2007). 
BESSIÈRE

mon absence $\gg$, , Courrier des Antipodes $\gg, ~ «$ Nouvelles Indes Galantes $\gg$, « Bicentenaire Kit », « Archipel Shopping », « Carnaval transatlantique $»^{7}$, ne sont pas des titres de chapitres, mais ceux, continus, de bandeaux (aux couleurs d'impression différentes), entrecroisés tout au long de l'ouvrage, en un jeu sur leurs positions dans les pages. Dans l'ordre qui vient d'être noté et suivant les indications que donne Butor dans la quatrième de couverture, ces titres renvoient à des espaces d'animaux, à la Colombie britannique, à l'Australie, à l'espace de l'opéra, aux États-Unis, à Singapour et Formose, et au Brésil et à Nice ${ }^{8}$. De plus, faute que ces titres soient repris dans le corps de l'ouvrage, seules des sortes de mentions courantes, placées au centre ou en bas des pages, donnent des noms de lieux, d'objets, de monnaie - autant de moyens d'identifier des espaces, simples points de repère dans le croisement continu des bandeaux. Ainsi, le livre de voyage est-il celui d'une série de voyages, présentés sans argumentation globale, celui d'une textualité segmentée - mime continu des séries de voyages, de fait, un vaste patchwork, de plus de 450 pages d'une impression fort serrée, qui invite le lecteur à lire selon cette discontinuité, à faire de sa lecture le mime des multiples orientations du voyage. Butor amplifie la disparité déjà manifeste dans Où et celle d'Un barbare en Asie, et la variation des identifications des scènes évoquées, hors de toute possibilité de totalisation.

Qu'il s'agisse de Michaux ou de Butor et quelle que soit l'étendue du voyage, aucun lieu n'est perçu en son entier, mais toujours relatif à un autre - ainsi du glissement d'un lieu à l'autre chez Michaux, de la contiguïté de tous les lieux chez Butor. Le voyageur est sans pouvoir sur le lieu, comme celui-ci l'est sur le voyageur ; il n'est jamais immergé dans l'espace, quels qu'en soient la densité et le pouvoir d'attraction, quelle qu'en soit l'inextricabilité - c'est là le thème du bandeau « Jungle » sur lequel s'ouvre Boomerang. Rendre compte de la perception de ce qui est dans l'espace est toujours un exercice double : de cet espace même et de son autre. De la dualité de tout voyage - voir ce que je vois, voir ce que je m'attends de voir - est indissociable la dualité de tout lieu du voyage - ce lieu est indissociable d'un autre lieu -, qui ouvre à l'amplification de toute perception et à des attentes du voyageur, qui ne sont plus nécessairement conventionnelles.

\footnotetext{
Remarquons que cette liste de titres n'apparaît que dans la table des matières et que les identifications synthétiques des espaces auxquels ils correspondent ne sont précisées qu'en quatrième de couverture.

8 Rappelons que Nice était la ville de résidence de Butor durant ces voyages. Cela est une des justifications du titre «Boomerang »: retour à l'espace domestique.
} 
III. Écriture littéraire du voyage, ses réflexivités, ses paradoxes, son degré zéro

Cette phénoménologie du voyage, exposée par Michaux et par Butor, joue des perceptions, assurément immédiates, et de la diversité de leurs lieux et de leurs moments. Ces perceptions sont, en conséquence, celles de multiples comportements sociaux, alors placés sous le signe d'un behaviorisme. Inévitable lorsqu'un étranger décrit une société qu'il ne connaît pas ou qu'il connaît peu, ce béhaviourisme se confond avec une sorte d'objectivation de ce qui est vu. Cette objectivation, associée aux réactions, jugements et discours du voyageur, engage un jeu de désignation spécifique. Le behaviorisme ne peut aller au-delà des citations des conduites qu'il objective - ces conduites sont telles qu'elles apparaissent. Les réactions du voyageur, aussi incertaines, discutables, personnelles qu'elles puissent sembler, ont pour limite ce behaviourisme : elles ne peuvent le défaire sauf à effacer les données qui sont les objets du compte rendu du voyage. Le behaviourisme impose une évidence : tout discours du voyageur est bien à propos de ce dont il parle ; il est bien une désignation. Aussi, les réactions personnelles du voyageur, fussent-elles négatives, sont également des moyens de l'objectivation et de la désignation.

Chez Michaux, chez Butor, le voyage, indissociable d'un point de vue behaviouriste, devient observation et constat des pratiques humaines, très largement comprises, toujours différentes, égales dans la série des voyages, également représentables et représentées. Cette objectivation, reprise par l'écrit, devient représentation objective. Les représentations issues de schèmes behaviouristes et lisibles selon ces schèmes sans que les perspectives propres $\mathrm{du}$ voyageur soient effacées, sont comparables par le fait-même de leur statut. Ainsi, les comparaisons explicites ou induites sont-elles constantes dans Un barbare en Asie. Ainsi, les bandeaux de Boomerang sont-ils, par leurs croisements, des suggestions de comparaisons. Ces proximités des scènes humaines et ces comparaisons, que dessine tout voyage dans les limites temporelles et spatiales de ses références, dans les limites de son propre développement, ont pour objets, les conduites et les arts qu'évoquent le compte rendu du voyage et ce qu'implique le behaviourisme : une constante intentionnalité humaine distribuée diversement, et qui a toujours des effets (Gell 1998) lisibles dans les réactions du voyageur. Ainsi, Un barbare en Asie peut-il être lu, à travers ses divers espaces, ses diverses scènes, comme la vaste exposition, explicite, implicite, des séries d'intentions humaines, qui apparaissent, à travers les conduites, en Asie. Ainsi, pour mettre en évidence la constance des intentionnalités humaines, Butor accompagne-t-il, dans Boomerang, ses scènes de voyage de références anthropologiques, ethnologiques - du Brésil aux ÉtatsUnis -, de références aux arts - long développement sur Duchamp -, et de 
descriptions de la nature. Ces descriptions font de la captation de l'homme dans la nature la figuration de l'insertion de tout être humain dans les réseaux des intentionnalités, arrière-plans de tous les objets, de tous les arts, de toutes les conduites, et constants. La nature devient l'interprétant des mondes humains. Boomerang s'ouvre sur le bandeau « Jungle »- la nature est, dans ce bandeau, l'interprétant des mondes humains et de leurs réseaux d'intentionnalités.

Cette référence à un réseau d'intentionnalités ne doit pas ici être lue comme une référence à une manière d'humanisme. Dans la reconnaissance des intentionnalités s'expose ce qui est recherché dans le voyage et ce que celui-ci fait constater : les divers engagements, en diverses cultures, des agents humains dans le monde, selon divers moyens, selon diverses formes, selon les corps des hommes, selon leurs paroles. C'est là la meilleure justification de l'usage du behaviourisme, la meilleure explication du voyage comme lecture du monde, et, en conséquence, ce qui permet la meilleure caractérisation du double jeu réflexif de l'écriture littéraire du voyage, et des paradoxes de l'écrivain et de l'écriture du voyage.

Jeu réflexif de l'écriture du voyage. Tout lieu peut être l'objet d'un jeu réflexif : observation, reconnaissance des intentionnalités, et mouvement inverse. À l'opposé des thèses sur la modernité et la surmodernité, aucun non-lieu n'est à identifier. Reconnaîtrait-on un non-lieu - par exemple, un aéroport comme l'indique Marc Augé (Augé 1992) -, qu'il faudrait encore le considérer comme un lieu : il est une construction et un signe humains et sociaux, voies de la reconnaissance des intentionnalités.

L'écriture littéraire du voyage reprend ce jeu réflexif. Elle porte un mouvement représentationnel initial, simple et inévitable : celui de la citation des noms de pays, de lieux, de leurs moments, de leurs aspects. Ce jeu a comme son propre pas au-delà : la chaîne des citations implique, en elle-même, la reconnaissance des intentionnalités humaines. L'écrivain voyageur découvre cette implication, et prend conscience de la possibilité - de l'attente - de cette reconnaissance. Les descriptions précises, qu'offrent les comptes rendus de voyage, déploient les lectures de ces intentionnalités, à travers les aspects des lieux, les gestes humains - importance des gestes de l'habileté en Chine, faut-il rappeler, qui permettent de définir toute une culture (Michaux 2016, 198) ${ }^{9}$, des gestes rituels chez Butor (rituels des Zunis dans $O \dot{u}$ ), qui mènent à des visions anthropomorphes de la nature, du soleil, et font de l'écriture du voyage une écriture ethnologique et même la transcription de documents ethnologiques

9 Notons qu'une interrogation abstraite à propos du pays étranger - qu'est-ce que l'idolâtrie en Inde ? - trouve sa réponse dans l'observation du corps : « L'Hindou a l'idolâtrie dans la peau » (Michaux 2016, 33). 
(Butor 1971, 340). C'est pourquoi l'écriture littéraire du voyage, qui ne peut être à la mesure du monde, se confond avec l'amplification de la perception des lieux et des conduites. Hors de cette amplification, elle serait la seule citation de la disponibilité des lieux du monde et des observations que permet le voyage. Par cette amplification, elle figure le jeu réflexif de l'observation et de la reconnaissance des intentionnalités. Remarquons que, dans Boomerang, les longues séries de noms de lieux fort divers - sans autre précision - prêtent à l'implication et l'attente de la reconnaissance des intentionnalités, une pertinence universelle.

Paradoxe de l'écrivain voyageur. Cette attente et cette reconnaissance d'intentionnalités supposent quaucun lieu ne soit vu comme radicalement étranger - il est autre -, que seul le voyageur soit ainsi vu- donc, disponible aux lectures des intentionnalités. Voir ainsi l'autre lieu n'abolit pas cependant l'identité et la situation du voyageur : celui-ci ne cesse de suivre l'autre, qui est dans son propre espace. Michaux note ce point dans sa préface de 1967 à Un barbare en Asie : «Débarquant là, en 31, sans savoir grand-chose, la mémoire cependant agacée par des relations de pédants, j'aperçois l'homme de la rue. Il me saisit, il m'empoigne, je ne vois plus que lui [...]. J'ai ce qu'il faut pour tout comprendre... à peu près. » Dans la section initiale et finale de Oì, « J'ai fui $\gg$, Butor dit les circonstances de son départ de Paris, de son retour, son prochain départ : chaque fois prévaut l'attente de l'autre - autre lieu, autres êtres humains. Cette attente commande, chez Butor, les premiers exercices de la vue, les constats initiaux du silence. Vision et silence font entrer dans la disponibilité au tout autre et suscitent des descriptions de paysages naturels ou d'espaces urbains, où n'apparaît aucune silhouette humaine. L'absence de toute silhouette humaine n'exclut pas l'implicite présence autre. Tout cela fait le paradoxe de l'écrivain : celui-ci, étranger, entre toujours dans un exercice de familiarisation face à l'autre, sans abandonner son état d'étranger.

Paradoxe de l'écriture. Le compte rendu de voyage participe entièrement de l'énonciation du voyageur. Cette énonciation est indissociable du lieu, de l'être humain autres. Aucune distance ne peut être perçue entre ces deux faces de l'énonciation parce que celle-ci va avec la reconnaissance des intentionnalités. C'est pourquoi l'écrivain voyageur peut regretter ce qu'il a écrit, et dire cependant que le compte rendu de voyage vaut par lui-même, qu'il est inaltérable. Ce compte rendu expose un état de la reconnaissance de l'autre et de l'énonciation du voyageur ; celles-ci restent unies aux moments et aux lieux des perceptions. Michaux note dans sa préface de 1945 à Un barbare en Asie : «Douze ans me séparent de ce voyage. Il est là. Je suis ici. On ne peut plus grand-chose l'un pour l'autre. [...] Il a vécu sa vie. » Remarquablement, Butor affirme à la fin de Où l'inaltérable du voyage, par lequel l'ailleurs devient un 
BESSIÈRE

« ici ». À partir de cet «ici », Butor écrit qu'il « hait » Paris et qu'il reviendra à Paris. Il y a là à la fois une interprétation de Paris selon la vision de Butor, vision du voyageur, et cette évidence : ne pas nier le lieu domestique confirme l'inaltérable de l'« ici » du voyage.

Ces remarques permettent de relire le paradoxe de l'écrivain. L’identité de celui-ci, fût-elle seulement celle de son statut d'écrivain, doit rester assurée sans elle, la lecture des intentionnalités des hommes et des femmes de la terre du voyage serait impossible. Cette identité peut être comme amoindrie, à peine citée, réduite au jeu de l'énonciation, en particulier chez Butor. Dans une vue rétrospective, elle apparaît, chez Michaux, entièrement circonstancielle, indissociable de la terre du voyage. Mais cet amoindrissement autorise l'amplification de la reconnaissance des intentionnalités.

Il est patent que le voyageur est en lui-même constant. Il est encore patent que le lieu du voyage est reconnu comme tel. Il est manifeste que l'écriture littéraire du voyage se donne, par l'organisation de ses évocations, comme telle. Tous ces caractères restent indissociables de l'énonciation auctoriale. Inévitable dans le compte rendu de voyage, elle ne peut faire loi - elle est selon les lieux, les temps, le behaviourisme, les intentionnalités. Quoi que l'écrivain voyageur ait pu imaginer de ce qu'il allait voir, quels quaient pu être ses visées et ses préjugés, la fonction précise de son énonciation - aller selon le droit de cité de l'autre, qui ouvre au constat des intentionnalités -, le statut de l'écriture, tel qu'il a été précisé, ramènent le compte rendu du voyage à la dualité initialement notée : voir ce qu'on voit, voir ce qu'on s'attend à voir. Ce retour a deux raisons. Si le voyage n'a pas de finalité pratique - découverte, pouvoir, connaissance, quête de quoi que ce soit -, s'il doit être dit hors de la seule énumération des lieux, il rapporte ce que fait inévitablement le voyageur : percevoir, constater et venir nécessairement à l'observation d'un behaviourisme, et à ceux-là qui sont impliqués, les agents humains, et à cela qui est impliqué, les signes et les objets. L'observation du behaviourisme est une observation inclusive de tout ce qu'une culture fait apparaître d'elle-même, y compris le plus connu, le plus convenu, et des effets que cette culture porte en elle-même pour ses propres agents. Le voyageur est contraint à une nouvelle attente, constante durant le voyage : celle de ce que peut faire entendre l'observation du behaviourisme. Cette nouvelle attente ne contredit pas nécessairement les attentes initiales du voyageur. Celles-ci, reprochées au touriste, portent souvent sur des caractéristiques largement connues ou sur des images reçues du pays étranger, et renvoient implicitement ou explicitement aux intentionnalités de l'autre, qui peuvent encore correspondre, pour le voyageur, à des vues conventionnelles de l'étranger. Ainsi, subsiste l'attente, renouvelée parce qu'elle est à l'étranger indissociable de l'observation. 
Voir ce que je vois, voir ce que je miattends à voir

Tout cela fait le degré zéro de l'écriture littéraire du voyage. Il reste à examiner ce qui fait spécifiquement de cette écriture une écriture littéraire.

\section{IV. Écriture du voyage, écriture littéraire}

Par littéraire, on peut comprendre ce discours qui présente certaines formes, certaines conventions, certains codes - tout cela est fort variable -, qui répondent chaque fois à une caractérisation de la littérature. Un tel type de discours n'impose pas, en conséquence, des règles constantes et n'est pas nécessairement utilisé dans les comptes rendus de voyage. Cette «indifférence » des comptes rendus de voyage à des traits reconnus pour littéraires n'exclut pas que tel compte rendu présente des formes complexes, des aspects expressément littéraires (poésie), sans que, pour autant, il offre, en lui-même, les moyens d'une caractérisation littéraire du type de celles qui viennent d'être citées.

Pour préciser en quoi l'écriture du voyage peut être une écriture littéraire, il faut considérer nouvellement les caractérisations « normées » de la littérature, quelles que soient ces normes ou les contre-normes qui en sont, de fait, indissociables. Envisageons abstraitement un objet littéraire qui répond manifestement aux caractères de la littérature, définis à tel moment. Ce discours se lit sur le fond d'autres discours ; par ses traits caractéristiques, il s'en distingue et fait question - question d'intelligibilité, d'intentionnalité - ; par cette question, il n'est pas dissociable des autres discours, qui, entendus comme du dehors, ainsi que le fait des discours des autres le voyageur, sont ce même type de question et la question du « distributed mind $\gg$, arrièreplan des intentionnalités. L'œuvre littéraire « normée », par les questions explicites qu'elle porte ou celles qu'elle appelle, à partir de sa lettre, est la figuration de la question dont les intentionnalités et le « distributed mind » suscitent l'inférence. Remarquons que le nominalisme littéraire - une œuvre déclarée littéraire par son auteur, par la critique, alors qu'elle ne présente pas de traits tenus pour littéraires - confirme ces remarques : discours isolé par sa seule identification, cette œuvre fait question comme le font les œuvres manifestement caractérisables comme littéraires. Elle est l'indication et le mime du questionnement qu'induit toute œuvre littéraire codée, et de celui qu'appellent les ensembles discursifs.

Revenons au compte rendu de voyage. Il peut certes être dit littéraire si son auteur se reconnaît d'écrivain, s'il porte de manifestes intentions littéraires. Plus essentiellement, il peut être caractérisé comme littéraire pour des raisons indissociables de son statut.

Le voyageur, par la dualité de son regard - je vois ce que je vois, je vois ce que je m'attends à voir -, isole des faits, des scènes du pays étranger. Le compte 
rendu de voyage ne cesse de présenter des isolements - les divers paysages et scènes qu'il retient -, se donne comme une somme d'isolements, et, en conséquence, comme un isolat, ainsi que l'œuvre littéraire est un isolat même si elle relève d'un nominalisme littéraire. C'est pourquoi le compte rendu est celui d'un aller et retour - ce mouvement qui, dans son actualité, est une transition et donc un isolat au regard des contextes du lieu d'origine et du lieu étranger. Aussi, ce compte rendu impose-t-il, de lui-même, le même type de question qu'impose l'œuvre littéraire.

Sont congruents avec ces jeux d'isolements et la question qu'ils portent l'organisation, le constructivisme et bien des détails du compte rendu - séries des observations, des appels d'inférence que portent les intentionnalités, notés soit directement au moyen du thème de la foule indienne, de l'artisan chinois dans Un barbare en Asie, soit indirectement au moyen de rappels ethnographiques dans Où et dans Boomerang. Avec ces jeux d'isolement et avec la question qu'ils portent, sont encore congruents les paradoxes de l'écriture, ceux des scènes des lieux étrangers et tous les moyens d'indiquer ou de faire penser une réflexivité. Est encore congruente la figure de l'écrivain voyageur. On a noté la fonction de désignation qu'ont les impressions positives et négatives du voyageur (Michaux), les méditations étrangères au voyage même, qui sont des manières d'extension des observations (Butor).

Tout cela constitue des constats qui s'imposent aux lecteurs. De plus, même si le compte rendu ne joue pas explicitement des effets du dépaysement, même s'il ne se veut pas un récit de la recherche de la terre explicitement inconnue, il est toujours et inévitablement une communication de l'ignorance communication de ce que ne peut savoir le lecteur, qui ignore les détails précis de la culture autre, du voyage de l'écrivain, des réactions de ce dernier. Par la communication de l'ignorance, le compte rendu impose à ses lecteurs, pour ce qui concerne sa textualité, les agents humains, les cultures évoquées et le voyageur, la même attente d'intelligibilité et d'intentionnalités que le voyage a suscité chez le voyageur. Au regard de ses lecteurs, le compte rendu non seulement rend compte du voyage, mais aussi en mime le statut : il place ses lecteurs dans une situation de lecture (d'observation) similaire à celle du voyageur.

Sans qu'il soit fait référence à des normes de la littérature, ce mime et cette similitude font le caractère littéraire de l'écriture du voyage, qui retrouve ainsi le questionnement qu'illustre toute œuvre littéraire, identifiée comme telle.

Outre tout ce qui vient d'être noté, la formalisation textuelle de l'espace illustre spécifiquement cette écriture/construction du compte rendu de voyage. Cette formalisation est directement lisible, dans Boomerang, dans Où : tout lieu devient textuellement une scène spectaculaire, organisée et dite 
de telle manière qu'elle dessine le mouvement de l'attention du voyageur celui-ci n'est pas cependant présenté comme captif de ce qu'il voit. Est ainsi ouvert le mouvement de l'observation à l'attente et la reconnaissance des intentionnalités. Cette formalisation textuelle est également manifeste dans $U n$ barbare en Asie : l'écriture de l'ouvrage, qui n'est pas commandée par la situation précise du voyageur en Chine - Michaux remarque que son état d'étranger est sans importance - est une libre écriture de l'espace, qu'elle ne cesse de segmenter et de cadrer. La complexité et l'étendue de cette formalisation, manifestes dans Un barbare en Asie et dans Boomerang, obligent à une lecture littérale qui fait revenir le lecteur à la question qu'est le compte rendu.

\section{V. Écriture, ultime réflexivité, ultime figure du voyageur}

L'écriture littéraire du voyage caractérise de manière bien spécifique le voyageur. Celui-ci voyage ; il n'est jamais, a-t-on noté, un voyageur souverain - ce défaut de souveraineté est indissociable de la dualité de la perception des lieux et des hommes, voir ce qu'on voit, voir ce qu'on s'attend à voir. Il dessine, dans son compte rendu, un monde global. Par ses observations, il est autant un observateur qu'il est un sujet produit par elles - un sujet constant, mais inscrit dans divers espaces. Le compte rendu de voyage note cela même.

L'écriture et l'organisation du compte rendu de voyage doivent allier l'égalité des lieux, l'égalité des sujets et le dessin de la continuité du voyage, quel que soit le nombre de ses séjours ; sans tout cela, la dualité de la perception du voyageur ne pourrait être présentée comme constante. Cette organisation doit aussi être d'une écriture suffisamment variée - un carnaval, selon une idée de Butor -, sans laquelle les lieux et leurs scènes n'apparaîtraient pas comme riches de spécificités et d'un égal droit de cité ; cet égal droit de cité suggère une cohésion textuelle du compte rendu. Aussi hétérogène qu'il soit dans ses évocations des lieux, celui-ci est, en conséquence, une continuité et un tout, sans qu'il dessine un espace supérieur, un espace englobant. Le seul espace qui puisse être conçu comme englobant est celui du monde ; mais aucun voyageur n'a vu l'ensemble du monde, sauf à être hors du monde, ou à user d'un moyen de transport qui le mettrait au-dessus de ce monde, dans cet espace que dessinerait un tour du monde en avion et qu'évoque Réseau aérien de Butor (Butor 1962). Par un jeu supplémentaire de son organisation, le compte rendu de voyage suggère cependant un substitut à un espace englobant : il prête aux mondes des voyages une ontologie spécifique; il les présente selon des emboîtements et des recouvrements partiels, ou les impliquent. Il permet ainsi de penser le monde des mondes, qui n'est pas un monde englobant, ce super-objet qui inclurait les mondes. Il faut redire Un barbare en Asie, Où et Boomerang. 
BESSIÈRE

Cette pensée d'un monde des mondes selon des emboîtements et des recouvrements multiples, remarquablement suggérée par la construction discontinue de Boomerang, renvoie à l'ultime jeu de réflexivité du compte rendu de voyage. Le voyageur aux multiples observations devient, lorsqu'il écrit, l'observateur des observations : il construit ainsi l'observation des manières dont les mondes sont distingués lorsqu'on passe de l'un à l'autre en les observant. Cette ultime observation dessine un tout dicible seulement par des différenciations, cependant corrélées - emboîtements, recouvrements. Que le voyageur, écrivant, écrivain, soit cet observateur le fait paradoxalement apparaître comme le produit d'un ensemble d'espaces et, en conséquence, comme un sujet définissable par leurs différenciations. Que Michaux voie, après la seconde guerre mondiale, Un barbare en Asie comme un texte caduc indique qu'il ne souhaite plus tenir cet ultime jeu réflexif pour actualisable. Il reprendra cependant une lecture du sujet, soumis à et produit de différenciations, dans ses expériences $\mathrm{du} \ll$ dedans ». Le lecteur, aujourd'hui, ne cesse de poursuivre avec le voyage et ses mondes, d'actualiser la pensée du monde de ces mondes et la figure d'un voyageur qui ne peut être un voyageur souverain, quelle que soit l'étendue de ses voyages. Par quoi l'écriture littéraire du voyage est enfin une heuristique du voyage.

\section{Jean Bessière}

jean.bessiere@sorbonne-nouvelle.fr

Université Sorbonne Nouvelle

FRANCE

\section{Références}

Augé, M. 1992. Non-Lieux. Une anthropologie de la surmodernité. Paris : Le Seuil.

Augé, M. 1997. L'impossible voyage. Le tourisme et ses images. Paris : Payot $\$$ Rivages.

Augé, M. 2003. Le temps en ruines. Paris : Galilée.

Botton de, A. 2002. The Art of Travel. New York: Vintage.

Butor, M.1978. Boomerang. Le génie du lieu 3. Paris : Gallimard.

Butor, M.1958. Le génie du lieu. Paris : Grasset.

Butor, M. 1962. Réseau aérien. Paris : Gallimard.

Butor, M. 1971. Où (avec accent grave barré, qu’on ne peut reproduire ici), Le génie du lieu 2. Paris : Gallimard.

Gell, A. 1998. Art and Agency: An Anthropological Theory. Oxford: Clarendon Press.

Leys, S. 2007. Michaux : un Belge en Asie. Commentaire, 117, 231-237. https://doi. org/10.3917/comm.117.0231

Michaux, H. 2016. Un barbare en Asie. Paris : Gallimard, 1933 ; édition revue et corrigée, Paris : Gallimard, 1976. Nous utilisons cette édition, telle qu'elle est publiée dans sa version électronique de la collection « L'imaginaire », 2016. 